\title{
ESTUDO EPIDEMIOLÓGICO DA PRESCRIÇÃO DE MEDICAMENTOS POTENCIALMENTE INAPROPRIADOS PARA IDOSOS NO MUNICÍPIO DE ALFENAS/MINAS GERAIS
}

Gabriella Stravini de OLIVEIRA ${ }^{1}$

Cláudio Daniel CERDEIRA ${ }^{2}$

Gérsika Bitencourt Santos BARROS ${ }^{1}$

\author{
${ }^{1}$ Departamento de Medicina, Universidade José do Rosário Velano, UNIFENAS-Alfenas. \\ 2Universidade Federal de Alfenas, UNIFAL-MG.
}

Recebido em: 01/03/2016 - Aprovado em: 10/03/2017 - Disponibilizado em: 01/07/2017

\begin{abstract}
RESUMO
Introdução: A alta prevalência de doenças na terceira idade acarreta em uso de um grande número de fármacos pelos idosos, sendo a prescrição de medicamentos a intervenção terapêutica mais comumente realizada. Diante disto, o impacto das prescrições exige a criação de novas estratégias, como a detecção de medicamentos potencialmente inapropriados (MPIs) para idosos. O medicamento é considerado inadequado para idosos quando os potenciais riscos são superiores aos benefícios (critérios de "Beers-Fick").Metodologia: Neste estudo observacional/transversal foram investigados dados provenientes dos prontuários dos idosos cadastrados no sistema único de saúde (SUS) do município de Alfenas, Minas Gerais. Para tanto, foram aplicados os critérios de "Beers-Fick". Resultados: Dos 384 prontuários analisados, foi constatado que68 prescrições (17,7\% dos prontuários) estavam em desacordo com os critérios de "BeersFick", apresentando no mínimo um MPI, sendo que alguns prontuários apresentaram prescrição de vários MPIs a um mesmo paciente.Os MPIscom maior frequência de uso entre os idososforam: amitriptilina, diazepan, nifedipina, sulfato ferroso,cimetidina, nitrofurantoína, doxasozina, metildopa, ciclobenzaprina e amiodarona.Conclusão: Como mostrado neste estudo, uma considerável e preocupante frequência de MPIsé prescrita em um município do Sul de Minas gerais. A utilização dos critérios de "Beers-Fick" pode prevenir as prescrições de MPIs aos idosos, em um contexto que abrange uma abordagem interdisciplinar.
\end{abstract}

Palavras chave: Medicamentos potencialmente inapropriados, Idosos, Prescrição

\begin{abstract}
Background: The high prevalence of age-related diseasesentails the use of a great number of drugs by elderly patients, further the prescription of drugs being a therapeutic intervention commonly performed. In view of this, the impact of prescriptions requires the creation of new approaches, such as the detection of potentially inappropriate medications(MPIs) in elderly. A drug is considered inappropriate for elderly patients when the potential risks outweigh the benefits ("Beers-Fick" criteria). Methods: In this observational/transversal study were investigated data from the medical records of elderly patients enrolled in the Unified Health System in Alfenas city, Minas Gerais (Brazil). Then, the "Beers-Fick" criteria were applied. Results: Of the 384 records analyzed, it was found that 68 prescriptions (17.7\%) were at odds with the "Beers-Fick" criteria, containing at least an MPI, and some records had prescriptions of various MPIs to a same patient. The MPIs with higher frequency of using among the elderly patients were: amitriptyline, diazepam, nifedipine, ferrous sulfate, cimetidine, nitrofurantoin, doxazosin, methyldopa, cyclobenzaprine, and amiodarone. Conclusion: As shown in this study, considerable and worrying frequencyof MPIs is prescribed in a southern municipality of Minas Gerais. The use of the "Beers-Fick" criteria can prevent MPIs prescriptionsamong elderly patients, in a context that includes an interdisciplinary approach.

Keywords: Potentially inappropriate medications, MIPs, Elderly, Prescription
\end{abstract}




\section{INTRODUÇÃO}

Atualmente, o Brasil encontra-se em um processo de transição demográfica, em que éobservado um aumento progressivo da população idosa, sendo a expectativa mundial para um cenário em que existirão mais idosos que crianças abaixo de 15 anos(PASCHOALet al., 2005; BRASIL, 2006).

O envelhecimento populacional é um desafio mundial a ser enfrentado, pois uma das mais sérias consequências é o predomínio de agravosde saúde crônicos (doenças crônico-degenerativas), que notoriamente impõe um importante impacto na sociedade brasileira, principalmente nos sistemas de saúde, exigindo serviços adequados ao cuidado em geriatria (BARDELet al., 2000; PASCHOALet al., 2005; VERAS, 2009).

A alta prevalência de doenças na terceira idade acarreta em um grande número de fármacos usados por parte deste público (KLOTZ et al., 2008, SILVAet al., 2012). A prescrição de medicamentos é a intervenção terapêutica mais comumente realizada por médicos. A preocupação com o impacto das prescrições para idosos exige a criação de novas estratégias para lidar com esse fenômeno, como a detecção de medicamentos potencialmente inapropriados, os MPIs (ROZENFELD, 2003; KLOTZ et al., 2008; GORZONIet al., 2012).
Neste contexto, alguns critérios para determinação os MPIs foram adotados, dentre os quais é destacado o de Beers (1997) mais tarde atualizado por Ficket al. (2003). Estes trabalhos se basearam em trabalhos publicados sobre medicamentos e farmacoterapeuticano envelhecimento, para definir uma lista de MPIs para pessoas com 65 ou mais anos de idade.

De acordo com esses critérios, um MPIindica que os riscos potenciais do medicamento são superiores aos benefícios potenciais que seriam atingidos com seu uso. Estes critérios não são indicativosde efeitos adversos dos medicamentos, mas determinam a inadequação do medicamento com base no seu risco potencial (BEERS, 1997; Ficket al., 2003; GORZONIet al., 2012).

No âmbito da atenção primária à saúde, acredita-se que iniciativas como a estratégia saúde da família (ESF), implementadas em PSFs, devam identificar e prevenir os resultados negativos associados à medicação, principalmente a redução de eventos adversos aos pacientes, bem como diminuir os gastos em saúde pública pelo uso inadequado de medicamentos (SECOLI, 2010;MASTROIANNI,2009).

Este estudopoderá contribuir no direcionamento de prescrições racionais de medicamentos para garantir maior segurança e qualidade de vida aos idosos. Além disso, poderá contribui sobremaneira ao campo da 
medicina geriátrica,por tratar-se de uma análise de uma amostra cujos dados não são encontrados na literatura especializada, havendo uma lacuna científica e social a ser preenchida. Assim, foi avaliada a prevalência do uso de MPIs entre os idosos atendidos pela atenção primária de saúde no município de Alfenas/MG, com identificação dos MPIs, segundo classe farmacológica.

\section{MÉTODOS}

O presente trabalho trata-se de um estudo de caráter observacional de natureza transversal, tendo sido analisados dados através de análises dos prontuários dos idosos cadastrados no SUSdo município de Alfenas, Minas Gerais. Para a composição da amostragem $(n)$, baseando-se na população total do município de Alfenas e na frequência estimada de idosos atendidos pelo SUS local, foi utilizado o cálculo do tamanho da amostra para proporção baseado na aproximação normal (FERREIRA, 2005), conforme mostradoa seguir:

$$
n=\frac{\left(Z_{\alpha / 2}\right)^{2} \times \hat{p} \times \hat{q}}{e^{2}}
$$

$\alpha=$ nível de significância; ${ }^{\alpha / 2}=$ quantil superior $\alpha / 2$ da distribuição normal padronizada; $^{p}=$ parâmetro da distribuição binomial; $q=1-p ; e=$ margem de erro fixada pelo pesquisador, portanto:

$$
\begin{aligned}
& n=\frac{(1,96)^{2} \times 0,5 \times 0,5}{0,05^{2}}=384 \\
& \alpha=0,05 ; Z_{\alpha / 2}=1,96 ; p=0,5 ;{ }^{q}=
\end{aligned}
$$

0,$5 ; e=5 \%$.Logo, o $n$ foi de 384 registros.

Para a realização deste estudo, os critérios de "Beers-Fick" foram aplicados. Esses critérios, por conseguinte, foram adaptados à farmacopeia brasileira, a fim de organizar e comparar os MPIs para os idosos analisados, independente do diagnóstico ou da condição clínica do paciente.O presente estudo foi previamente aprovado pelo Comitê de Ética em Pesquisa envolvendo seres humanos da UNIFENAS/Alfenas-MG (CAAE: 41215015.1.0000.5143).

\section{RESULTADOS}

Neste estudo foram analisados 384 prontuários de idosos acima de 60 anos residentes no município de Alfenas, Minas Gerais, no ano de 2015. A média da faixa etária dos pacientes analisados foi 73 anos. Dos 384 prontuários analisados, 68 prescrições continham, no mínimo, 1 MPI para os idosos. Constatou-se que, $17,7 \%$ dos prontuários analisados, estavam em desacordo com os Critérios de "Beers-Fick".

Os MPIs mais prescritos para os idosos avaliados, seguindo os critérios de "Beers-Fick", foram: nifedipina (prescrição deste fármaco apareceu em 28 prontuários), 
amitriptilina (constante em 17 prontuários), diazepan (prescrita em 14 prontuários), amiodarona (6), sulfato ferroso (7 prescrições), nitrofurantoína (4 prontuários), doxazosina (4), metildopa (3prontuários), ciclobenzaprina (3) e cimetidina(2) (Figura 1abaixo).

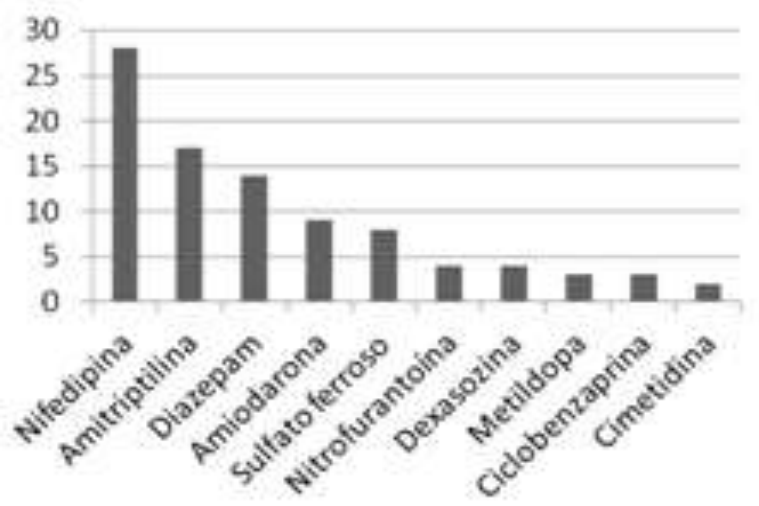

Figura 1 Distribuição do quantitativo de medicamentos potencialmente inapropriados (MPIs) prescritos para os pacientes em estudo, no município de Alfenas-MG, 2015

Em seguida, foi analisada a relação de MPIsem cada prescrição (Tabela 1abaixo) e obteve-se o seguinte resultado: 50 prescrições $\begin{array}{lll}\text { continham apenas } & 1 \mathrm{MPI}\end{array}$ prescrições2MPIs e 3 prescrições 3 MPIs. Assim, aproximadamente $26,4 \%$ das prescrições com esses tipos de fármacos continham 2 ou mais MPIs.

Tabela 1 Proporções de números de medicamentos potencialmente inapropriados (MPIs) usados por idosos por prescrição

\begin{tabular}{cc}
\hline $\begin{array}{l}\text { Número } \\
\text { de MPIs }\end{array}$ & Total de prescrições \\
\hline 1 & 50 \\
2 & 15 \\
3 & 3 \\
\hline
\end{tabular}

Verificou-se também o número de doses diárias por cada medicamento tomado pelos pacientes em questão. Com relação à amitriptilina, 6 prontuários receitaram uma dose, 3 prontuários, 2 doses e 8 prontuários não relataram. O diazepam foi receitado uma vez ao dia por 8 prontuários e 6 não registraram. A nifedipina foi tomada apenas uma vez ao dia segundo relatado em3 prontuários, 2 vezes em 9 prontuários e 3 vezes em 5 prontuários; 11 não registraram as dosagens. O sulfato ferroso foi administrado uma vez ao dia para 2 pacientes e 2 vezes para 3 pacientes; 2 prontuários não relataram. A cimetidina foi tomada 1 vez por 1 idoso e 2 vezes por outro idoso. A nitrofurantoína foi administrada 3 vezes para 3 pacientes, sendo 1 vez não registrada. A doxasozina foi receitada 1 vez ao dia em 2 prontuários, 2 vezes em 1 prontuário e 1 dosagem não foi relatada. Sobre a metildopa, administrou-se 2 vezes ao dia em 2 prontuários e 3 vezes ao dia em 1. Ciclobenzaprina não foi registrada em nenhum prontuário, sendo o total de 3 . Por fim, amiodarona foi administrada em 6 prontuários, 1 vez ao dia.

\section{DISCUSSÃO}

Ao se analisar os MPIs, constata-se que o fármaco prescrito com maior frequência, na população estudada, foi o que atua no sistema cardiovascular (Figura 1). 
Similarmente, este é o padrão de uso geralmente utilizado por idosos do país como relatado em alguns estudos (LIMA, 2008; CARVALHO, 2007).

Uma revisão sistemática de medicamentos utilizados pelos idosos deve fazer parte de todos os serviços de saúde ofertados no país, inclusive na atenção primária de saúde. A alta prevalência de doenças crônico-degenerativas eo grande número e o continuouso de fármacos entre os idosos causade duas a três vezes mais interações medicamentosas e reações adversas em idosos do que em adultos jovens, além dealterações na farmacocinética, farmacodinâmica e surgimento de reações adversas(DOUCETet al., 1996; MOSEGUIet al., 1999; CORSONELLOet al., 2010).

Prévios estudos têm relatado que o amplo uso de MPIs em instituições de longa permanência, ambulatórios, hospitais e comunidade variam na frequência de 10,4\%(ROZENFELD et al., 2008) a $48 \%$ (BALDONI et al., 2014). Portanto, a média de $17,7 \%$ encontrada nesse estudo está nesta faixa descrita. A variação dos valores encontrados pode se dar pelas diferentes formas de delineamento dos estudos, que apresentam amostras e contextos diferentes. Além disso, podem-se levar em conta os distintos perfis clínicos e sociais de cada localidade estudada.
O nifedipino, bloqueador de canal de cálcio, foi o medicamento mais utilizado pelos idosos avaliados neste estudo, em cerca de $40 \%$ das prescrições com MPIs. Esse fármaco é estudado como MPI pelo fato de causar vasodilatação, o que favorece maior risco de hipotensão e constipação, sendo assim, um grande risco a saúde do idoso.

Em seguida, foram mais utilizados amitriptilina e diazepam. Estes são medicamentos com ação no sistema nervoso central, um antidepressivo tricíclico e um benzodiazepínico, respectivamente, com potencial de sedação, ansiolítico, relaxamento muscular e anticonvulsivante. Ambos com risco potencial quando utilizados na terceira idade, devido ao fato que eles têm meia-vida prolongada, gerando risco de queda e fratura, o que explica a inclusão destes como MPIs.

A frequência expressiva de uso de diazepam, relatada em estudos brasileiros, incluindo o SABE (CARVALHO, 2007), pode estar relacionada ao seu baixo custo e disponibilidade, dado que é distribuído pelo SUS. Além disso, é um dos benzodiazepínicos mais frequentemente prescritos para tratar a insônia, condição frequentemente observada em idosos, devido à alteração do sono (NOIA et al., 2012).

Outro medicamento muito utilizado pelo grupo estudado foi $\mathrm{o}$ antiarrítmicoamiodarona. Esse medicamento sugere efeitos tóxicos ao idoso, assim seu uso 
por idosos requer o acompanhamento, recomendando-se o início do tratamento com dosagem mínima (SEMLA et al., 2008).

Neste estudo, aoserem seguidos os critérios de "Beers-Fick", algumas limitações são consideradas (SOARES et al., 2011). Primeiramente, a frequência de MPIs pode ter sido subestimada, pelo fato de apenas ser utilizado tais critérios, como listados. Ainda, a relação de MPIs tem como desvantagem a inflexibilidade em se falando de diferenças entre indivíduos.

Ainda, esses critérios não incluem algumas situações, como subdosagem do medicamento e a classe dos fitoterápicos, só abrangendo as prescrições equivocadas. Há também, dúvidas sobre listar algumas classes de fármacos, como amitriptilina, largamente utilizada em idosos(GALLANGHER et al., 2006).Esse fármaco não é completamente contraindicado à faixa etária estudada (O’MAHONY et al., 2008).

Por outro lado, a utilização dos critérios de "Beers-Fick", possui algumas vantagens, sendo útil em estudos farmacoepidemiológicos em vários tipos de populações, também sendo útil para fins educacionais.

Pode se dizer que há uma necessidade em adquirir condutas viáveis na diminuição da prescrição de MPIs aos idosos, consequentemente reduzindo as reações adversas.Neste contexto, investir na educação médica e em melhores hábitos de prescriçõessão essenciais.

\section{CONCLUSÃO}

A avaliação quanto às prescrições de MPIs para pacientes na terceira idadetêm grande valor epidemiológico, uma vez que reações adversas podem acarretar em sérias complicações aos pacientes. Portanto, esse é um grupo que requer atenção especial por correrem o risco de consequências negativas quanto ao uso de MPIs.

Assim, pode-se dizer que a utilização dos critérios de "Beers-Fick" pode prevenir as prescrições de fármacos inadequados aos idosos. Além disso, o uso de medicamentos em idosos é uma questão que abrange uma abordagem interdisciplinar. Portanto, médicos, farmacêuticos, enfermeiros, pacientes e familiares devem trabalhar em conjunto para melhorar a orientação quanto àconduta racional do uso de medicamentos,visando uma diminuiçãodo número de prescrições de MPIs aos idosos. 


\section{REFERÊNCIAS}

BALDONI, A.D.; AYRES, L.R.; MARTINEZ, E.Z.; DEWULF, N.D.; SANTOS, V.; PEREIRA, L.R. Factors associated with potentially inappropriate medications use by the elderly according to Beers criteria 2003 and 2012.Int J Clin Pharm, v. 36, n. 2, p. 316-324, 2014.

BARDEL, A.; WALLANDER, M.A.; SVARDSUDD, K. Reported current use of prescription drugs and some of its determinants among 35 to 65-year-old women in mid- Sweden: a population-based study.J ClinEpidemiol, v. 53, n. 6, p. 637-43, 2000.

BEERS, M.H. Explicit criteria for determination potentially inappropriate medication use by elderly.ArchInternMed, v. 157, p. 1531-36, 1997.

CARVALHO, M.F.C. A polifarmácia em idosos no Município de São Paulo: Estudo SABE- Saúde, Bem-estar e envelhecimento.Dissertação de mestrado,Faculdade de Saúde Pública da USP, 2007.

CORSONELLO, A.; PEDONE, C.; INCALZI, R. A. Agerelatedpharmacokineticandpharmacodynamic changesandrelatedriskof adverse drugreactions.Curr Med Chem,v. 17, p. 571584,2010 .

DOUCET, J.; CHASSAGNE, P.; TRIVALLE, C; LANDRIN, I; PAUTY, M.D; KADRI N, et al. Drug-drug interactions related to hospital admissions in older adults: a prospective study of 1000 patients.J AmGeriatrSoc,v. 44, n. 8, p. 944-948, 1996.

FERREIRA, D. F. Estatística Básica. $1^{a}$ ed., Editora Ufla, Lavras, 2005.

FICK, D. M.;et al. Updating the Beers Criteria for potentially inappropriate medication use in older adults: results of a US consensus panel of experts.Arch Intern Med., v. 163, p. 2716-2724, 2003.

GALLANGHER, P.; BARRY, P.;

O'MAHONY, D. Inappropriate prescribing in the elderly.J Clin Pharm Ther, v. 32, n. 2, p. 113-121, 2007.

GORZONI, M. L.; FABBRI, R. M. A.; PIRES, S. L. Potentially inappropriate medications in elderly. Rev. Assoc. Med. Bras., v.58, n.4,p. 442-446, 2012.

KLOTZ, U.; MÖRIKE, K.; SHI, S.The clinical implications of aging for rational drug therapy.Eur J ClinPharmacol., v. 64, p. 183-199, 2008.

LIMA, M.G. Fatores associados aos gastos com medicamentos utilizados por aposentados e pensionistas idosos em Belo Horizonte/MG. Tese de doutorado, Faculdade de Farmácia da Universidade Federal de Minas Gerais, 2008.

MASTROIANNI, P. C. Análise dos aspectos legais das prescrições de medicamentos. Rev. Ciênc. Farm. Básica Apl., v. 30, p. 173-179, 2009.

BRASIL, MINISTÉRIO DA SAÚDE. Secretaria de Atenção à Saúde. Departamento de Atenção Básica. Envelhecimento e saúde da pessoa idosa. Série A. Normas e Manuais Técnicos. Cadernos de Atenção Básica, n. 19. Brasília (DF); 2006.

MOSEGUI, G. B.; ROZENFELD, S.; VERAS, R. P.; VIANNA, C.M. Avaliação da qualidade do uso de medicamentos em idosos. Rev Saude Publica, v. 33, n. 5, p. 437-444, 1999.

NOIA, A. S.; SECOLI, S. R.; DUARTE, Y. A.; LEBRÃO, M. L.; ROMANO-LIEBER, N.S. Factors associated to the use of psychotropic drugs by community-dwelling 
elderly in São Paulo City. Rev Esc Enferm USP,v. 46, p. 38-43, 2012.

O'MAHONY, D.; GALLANGHER, P.F. Inappropriate prescribing in the older population: need for new criteria. Age Ageing,v. 37, n. 2, p. 138-41, 2008.

PASCHOAL, S.M.P.; SALLES, R.F.N.; FRANCO, R.P. Epidemiologia do envelhecimento. In: Carvalho Filho ET, Papaléo Neto M. Geriatria fundamentos, clínica e terapêutica. 2.ed.São Paulo: Atheneu; p. 19-34, 2005.

ROZENFELD, S.; FONSECA, M.J. M.; ACURCIO, F.A. Drug utilization and polypharmacy among the elderly: a survey in Rio de Janeiro City, Brazil. Rev Panam

Salud Pública,v. 23, n. 1, p.34-43, 2008.

ROZENFELD, S. Prevalência, fatores associados e mau uso de medicamentos entre os idosos: uma revisão. Cad Saúde Pública, v. 19, p. 712-724, 2003.

SECOLI, S.R.Polifarmácia: interações e reações adversas no uso de medicamentos por idosos.RevistaBrasileiraEnfermagem, v. 63, n. 1, p. 136-40, 2010.

SEMLA, T.; BEIZER, J. L.; HIGBEE, M.D. Geriatric dosage handbook: including clinical recommendations and monitoring guidelines.13th Ed. Hudson: LexiComp; 2008.

SILVA, A.L.; RIBEIRO, A.Q.; KLEIN, C.H.; ACURCIO, F.A. Utilização De

Medicamentos Por Idosos Brasileiros, De Acordo Com A Faixa Etária: Um Inquérito Postal.Caderno de Saúde Pública, v. 28, n. 6, p. 1033-1045, 2012.

SOARES, M.A; FERNANDEZ, L. F.; CABRITA, J.; MORAIS J.Tools to evaluate potentially inappropriate prescription in the elderly: a systematic review. Acta Medica Portuguesa, v. 24, n. 5, p. 775-84, 2011.
VERAS, R. Envelhecimento populacional contemporâneo: demandas, desafios e inovações.Revista de Saúde Publica, v. 43, n. 3, p. 548-554, 2009. 